\title{
ENTRAINER SELECTION FOR THE SYNTHESIS OF FATTY ACID ESTERS BY ENTRAINER-BASED REACTIVE DISTILLATION
}

\author{
M.C. de Jong ${ }^{1}$, A.C. Dimian ${ }^{2}$, N.J.M. Kuipers ${ }^{1}$ and A.B. de Haan ${ }^{1}$ \\ ${ }^{1}$ Separation Technology, University of Twente, P.O. Box 217, 7500 AE Enschede, \\ The Netherlands, E-mail: m.c.dejong@utwente.nl \\ ${ }^{2}$ HIMS, University of Amsterdam, Nieuwe Achtergracht 166, 1018 VW Amsterdam, \\ The Netherlands
}

\begin{abstract}
Residue curve maps, flash simulations and column simulation are used to investigate the effect of various entrainers with different polarity an esterification with isopropanol through Entrainer-based Reactive Distillation. Non-polar solvents are predicted to be good entrainers. For a more quantitative selection of entrainers further research concerning the thermodynamics is needed.
\end{abstract}

KEYWORDS: reactive distillation, entrainer, phase equilibria, fatty acids

\section{INTRODUCTION}

Fatty acid esters are natural-based chemicals used among other things in cosmetics, plastics and surfactants. Nowadays the fatty esters are produced in batch reactors with use of strong acids like sulphuric acid. Their production processes involve costly separations, large energy consumption and polluting by-products. Because of equilibrium limitations high conversions can be only obtained by using a large excess of alcohol. For a more competitive process it is preferable to produce fatty esters in a continuous way, at higher yields, in multi-product equipment, using a heterogeneous catalyst.

Reactive distillation is considered a promising technique because the integration of reaction and separation in one unit means significant savings in equipment and operation costs. One of the most known reactive distillation processes is the Eastman Chemical Company's methyl acetate process [1]. Also the esterification of other alcohols has been extensively investigated, but information on the esterification of long chain carboxylic acids such as fatty acids by reactive distillation is scarce. A few processes with various fatty esters and alcohols are described [2-4]. All refer to a homogeneous catalyst which causes pollution of the product. Steinigeweg and Gmehling [5] describe a process using a heterogeneous catalyst. They investigated the esterification of decanoic acid with methanol. The problem of all above processes is that the ester is not obtained in pure form such that further purification is necessary. Besides that, the alcohol, except for methanol, forms an azeotrope with water what is a disadvantage for realising higher yields. Omota et al. [6, 7] studied the feasibility of a single column process using a heterogeneous catalyst for the esterification of lauric acid with 2-ethyl-hexanol and methanol. They found that it is possible to obtain pure fatty acid ester in a single column process. Both esters can be produced in the same set-up, but under different operating conditions. 
However, problems can occur because of the product purity is highly sensitive to the reflux ratio. A promising way to improve this is by Entrainer-based Reactive Distillation based on solid catalysts [8].

In Entrainer-based Reactive Distillation in situ separation is used to improve the yield of reaction whereas an entrainer feed is added to make the separation feasible by selectively increasing the relative volatility of one of the products. Entrainer-based Reactive Distillation promises to be advantageous for the synthesis of fatty acid esters. The entrainer increases the relative volatility of water (by-product) compared to the alcohol (reactant), such that during the reaction the water can be continuously removed by distillation. In this way the chemical equilibrium is shifted such that higher conversions can be obtained. Preferably different esters will be produced in the same set-up (multiproduct design), possibly using the same entrainer which will be beneficial towards the investment costs. In Figure 1 the flowsheet of the desired process is given, in which RS stands for Reactive Section and DS for Distillation Section. This paper concentrates on the distillation section (DS) and the decanter (D), which primarily determine the selection of a suitable entrainer.

Besides acceptable as impurity, a suitable entrainer should fulfill the following criteria:

1. It should increase the relative volatility of water compared to alcohol, such that water can be removed over the top and the reaction equilibrium shifts to the product side.

2. It should show an immiscibility region with water, such that the distillate can be separated by decanting in a liquid entrainer phase and an aqueous phase.

3. It should have low solubility of entrainer in water, such that no further purification is necessary.

4. It should have low solubility of water in entrainer, such that the entrainer can be used as a recycle.

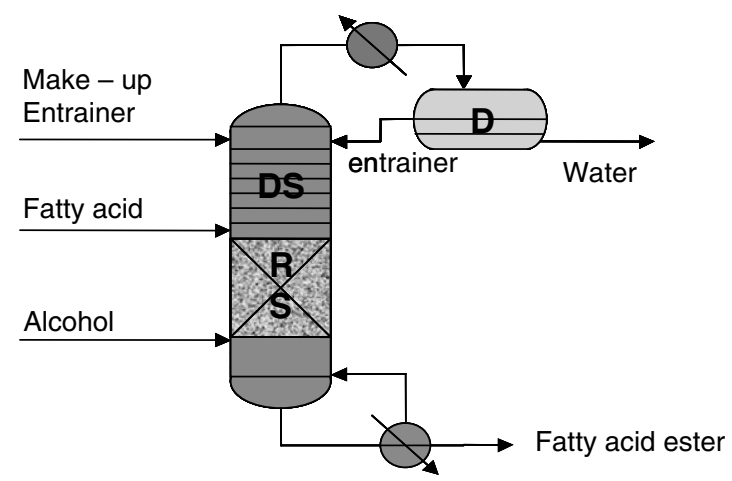

Figure 1. Conceptual flowsheet for entrainer-based reactive distillation synthesis of fatty acid esters 
The objective of this work is to investigate the effect of various entrainers with different polarity on the above criteria for a typical esterification such as that of lauric acid with isopropanol through Entrainer-based Reactive Distillation. Solvents with increasing polarity (Hexane, Cyclohexane, Benzene, Di-isopropyl ether and Isopropyl acetate) are studied as possible entrainers. For each criterion at least two techniques are available for entrainer selection:

- Residue curve maps provide information on criteria 2 till 4.

- Flash simulations provide information on criterion 1.

- Column simulations provide information on criteria 1 till 4.

Results are obtained by Aspen Plus ${ }^{\odot}$ and Aspen Split $^{\odot}$ simulations using the modified Unifac Dortmund property model.

\section{RESIDUE CURVE MAPS}

Usually residue curve maps are used to examine the feasibility of a certain separation: for a certain feed they show which residue and distillate composition can be achieved [9-11]. Normally these are used for validating the effects for a conventional distillation column including a reboiler and condenser.

However, in Entrainer-based Reactive Distillation the separation takes place in the upper part of a column (DS), while the reboiler is placed below the reactive section (RS). In this situation simulation results do not correspond with the residue curve map theory; distillation boundaries and residue curves can be crossed. So, in Entrainer-based Reactive Distillation separations can be achieved which are not possible in conventional or reactive distillation. Stéger et al. [12] also confirm that when a distillation column exists of different sections the residue curve map theory cannot be used. They studied batch extractive distillation in which there is a matter of an extractive and a rectifying section. Because there are two sections in the column, the composition profile consists of two parts and thus is a residue curve map not sufficient for studying the feasibility.

Though residue curve maps cannot be used for a quantitative analysis in this case they can be used for a qualitative analysis. The residue curve maps show for all studied solvents, that there is a liquid-liquid immiscibility for water and entrainer. For a known vapour composition the residue curve map can show the compositions of the liquid phases which occur after condensation.

In Figure 2 the residue curve map made with Aspen $\mathrm{Split}^{\odot}$ for benzene as entrainer is given as a typical example, in which the azeotropes are indicated with diamonds, the ternary heterogeneous azeotrope splits in an organic phase $o$ and aqueous phase $a$. For the mentioned criteria benzene seems to be a good candidate entrainer: There is a large immiscibility region between entrainer and water, which is close to both the water and entrainer vertex whereas the liquid-liquid tie-lines are orientated towards the water vertex. This means that after condensation and decanting the aqeous phase almost consists of pure water while the entrainer phase only shows a relative low water content. 


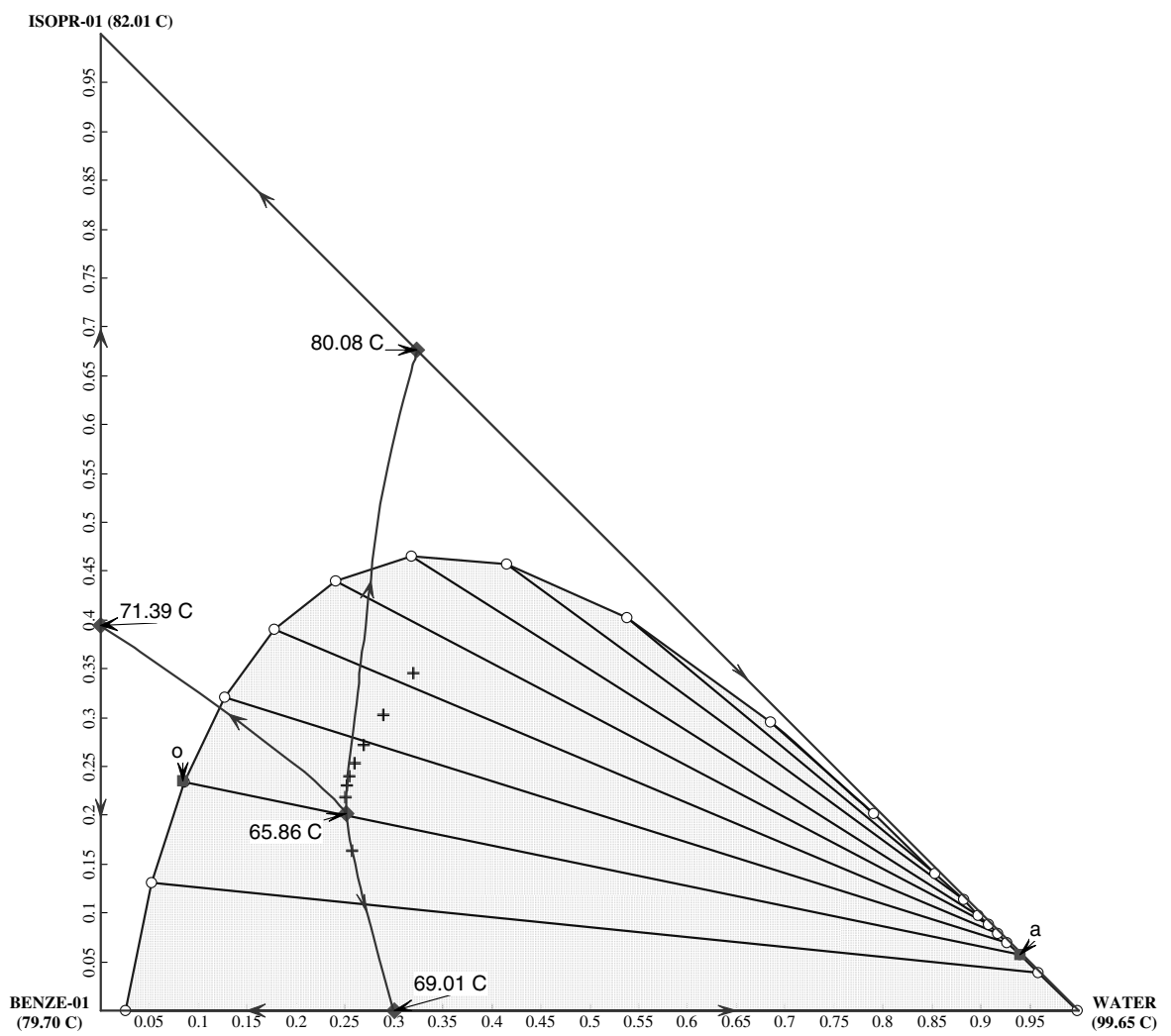

Figure 2. Residue curve map water-isopropanol-benzene at atmospheric pressure and boiling point of the mixture as simulated with Aspen Plus ${ }^{\odot}$ and Aspen Split $^{\mathcal{O}}$ $\checkmark=$ Azeotrope,$-=$ Liquid-liquid envelope $-=$ Liquid-liquid tie-lines, $o=$ organic phase, $\mathrm{a}=$ aqueous phase

The residue curve map of hexane shows an immiscibility region and tie-lines somehow similar to benzene. The immiscibility region of cyclohexane is situated closer to the entrainer vertex, which means that the entrainer phase contains less water than in case of benzene. But the tie-lines are not pointing to the water vertex, which means that the aqeous phase probably would contain alcohol and entrainer. In contrast, the tie-lines of Di-isopropyl ether and Isopropyl acetate do point to the water vertex but the immiscibility region is not situated close to the entrainer vertex, thus the entrainer phase will also contain more water. So far, it is not possible to draw final conclusions from this because at this stage it is not known which criterion is more important; a pure 


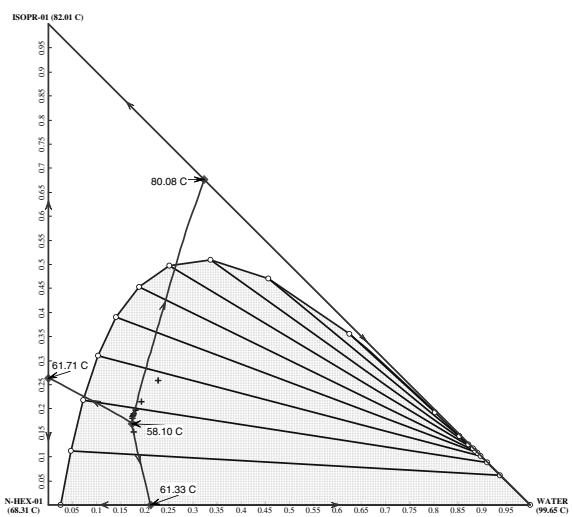

Hexane

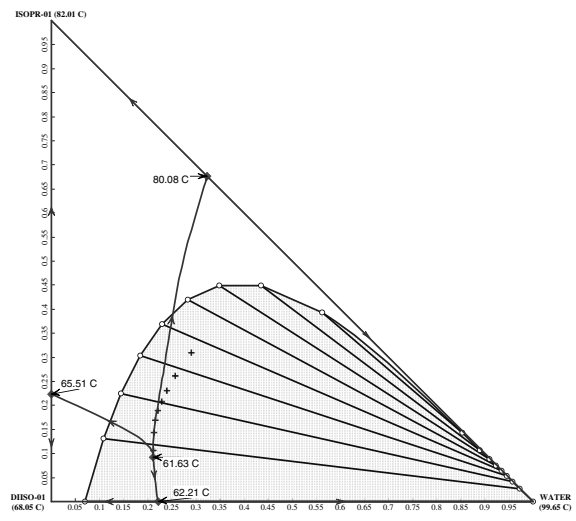

Di-isopropyl ether

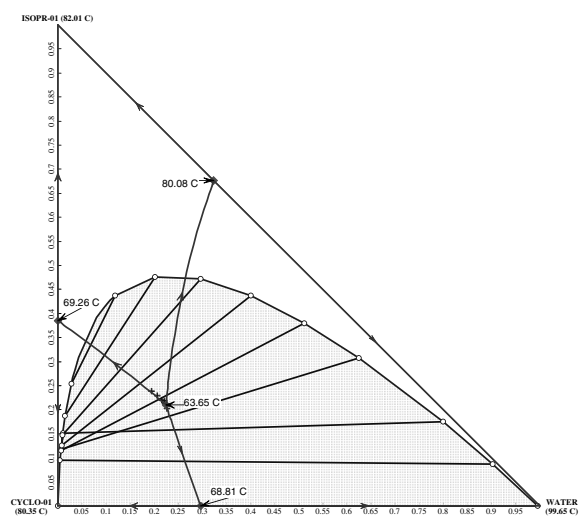

Cyclohexane

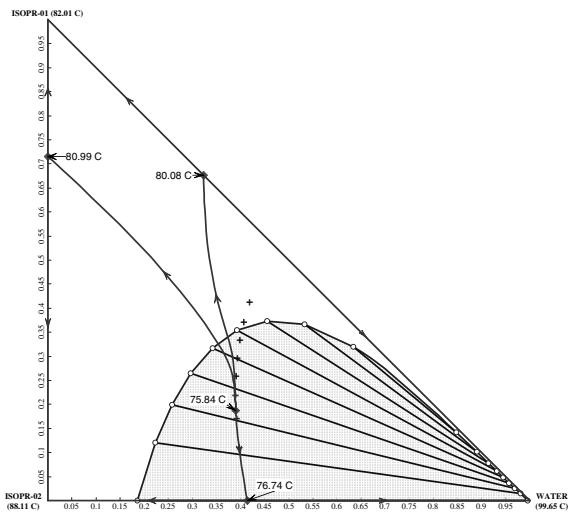

Isopropyl acetate

Figure 3. Residue curve maps for various entrainers for the water-isopropanol system as simulated with Aspen Plus ${ }^{\odot}$ and Aspen Split ${ }^{\odot}$.

water phase or a pure entrainer phase. The composition of the top vapour cannot be predicted from the residue curve map. Therefore, the exact compositions of the water and entrainer phase cannot be known. To investigate the exact effect of different entrainers, column simulations should be performed.

\section{FLASH SIMULATIONS}

In Aspen Plus ${ }^{\mathcal{C}}$ flash simulations were done in which the vapour-liquid equilibrium is calculated at atmospheric pressure and boiling point of the mixture. Starting point was an isopropanol-water mixture with azeotropic composition (68 mol\% ipa, $32 \mathrm{~mol} \%$ 
water) to which entrainer was added till a three-phase system (vapour-liquid-liquid) was reached. In Figure 4 the relative volatility $(\alpha)$ is set out against the mol fraction of entrainer. $\alpha$ is defined as the relative volatility of water compared to isopropanol:

$$
\alpha=\left(\mathrm{Y}_{\mathrm{H}_{2} \mathrm{O}} / \mathrm{X}_{\mathrm{H}_{2} \mathrm{O}}\right) /\left(\mathrm{Y}_{\mathrm{IPA}} / \mathrm{X}_{\mathrm{IPA}}\right) .
$$

Results show that for all of the studied entrainers the relative volatility of water increases when entrainer is added and that at certain compositions a three-phase system exists. It can be seen that the more non-polar solvents increase the relative volatility faster but also that a three-phase system is reached at lower entrainer mol fractions, see Figure 4a. If the starting point is an isopropanol-water mixture with a water content of $10 \mathrm{~mol} \%$, which probably will be the case in the distillation section of the column, only cyclohexane is predicted to reach a three-phase system, see Figure $4 \mathrm{~b}$. With a water content of $10 \%$, the relative volatility will increase more than for a water content of $32 \mathrm{~mol} \%$. This is also confirmed by the binary diagram of water and isopropanol, which contains a minimum boiling azeotrope at $32 \mathrm{~mol} \%$ water. Below the azeotropic point the relative volatility of water is higher.

The results are not in accordance with expectations. It is expected that the trend of the relative volatility for all solvents will be the same. While adding entrainer, the relative volatility will increase gradually. If a three-phase system is not reached a maximum relative volatility exists. When more entrainer is added the relative volatility will remain the same.
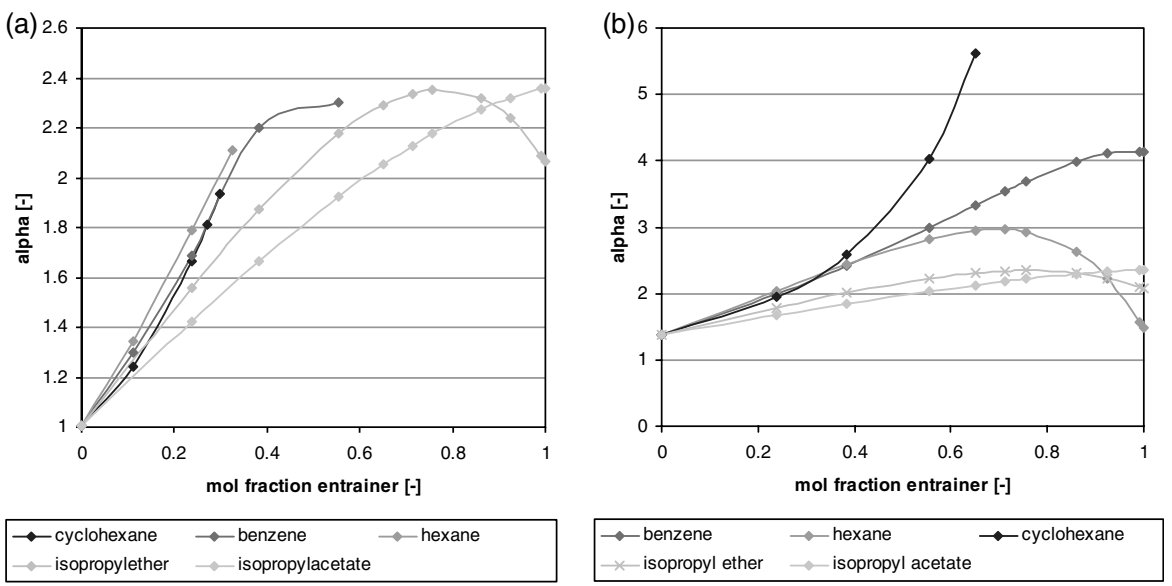

Figure 4. Relative water volatility regarding to isopropanol as function of mol fraction added entrainer till three-phase system is reached. (a) Water:isopropanol 0.32:0.68 (b) Water:isopropanol 0.1:0.9 
The deviation from these expectations may be caused by inaccurate predictions. The results are based on calculations with Unifac-Dortmund, which is a group contribution method. Activity coefficients are calculated from group interaction parameters, which are fitted from a large database of experimental data. The inaccurate predictions may be due to the fact that this database contains mostly vapour-liquid data and few liquidliquid data. Therefore it is preferable to use a thermodynamic model based on liquidliquid experimental data.

Hefter [13] evaluates experimental solubility data of cyclohexane in water and vice versa gathered from various authors. No data has been recommended for both solubilities. For the solubility of water in cyclohexane multiple data sets are available but these are not consistent. Only for the solubility of cyclohexane in water at $298 \mathrm{~K}$ sufficient data are available to make an evaluation. The solubility of cyclohexane in water is very low and therefore hard to measure. Therefore it is better to look at a three component system; when some alcohol is added more cyclohexane can solute in water. Because lower alcohols are used in the production process, these are taken as third component. In literature [14-19] data can be found for methanol, ethanol, propanol and isopropanol. Aspen should be used to regress NRTL parameters from these data. When this is done, the thermodynamica can be described more accurate as well as the simulations would give more accurate results.

\section{COLUMN SIMULATIONS}

In Aspen Plus the distillation section was simulated in a column without reboiler, see Figure 6. The isopropanol-water mixture enters the column at the bottom as a vapour. The entrainer enters at the top as a liquid. The effect of adding different amounts of entrainers was simulated while the alcohol-water feed was held constant. To distinguish between the effect on the separation by distillation and the separation by condensation, the reflux was not closed.

In Figure 7 the compositions of isopropanol and water as a pseudo-binary (in calculating the compositions the entrainer is left out of consideration), mixture are given for different amounts of entrainer added. It can be seen that after a certain amount of entrainer the bottom composition is constant while the top composition still improves. There is a maximum amount of entrainer because of the column operation window.

In Table 1 the results of the column simulation for the most preferable case (lowest alcohol content in top at lowest water content in bottom) are given for various entrainers. For almost all studied entrainers this refers to the maximum entrainer amount as limited by the operation window of the column.

It can be seen that the more non-polar solvents force more water to the vapour phase but also that a small amount of alcohol is also taken. However, the concentration of entrainer in the bottom product and the total amount of entrainer that is needed are important. In case of the non-polar solvents more entrainer can be used which results in a higher entrainer concentration in the bottom. 


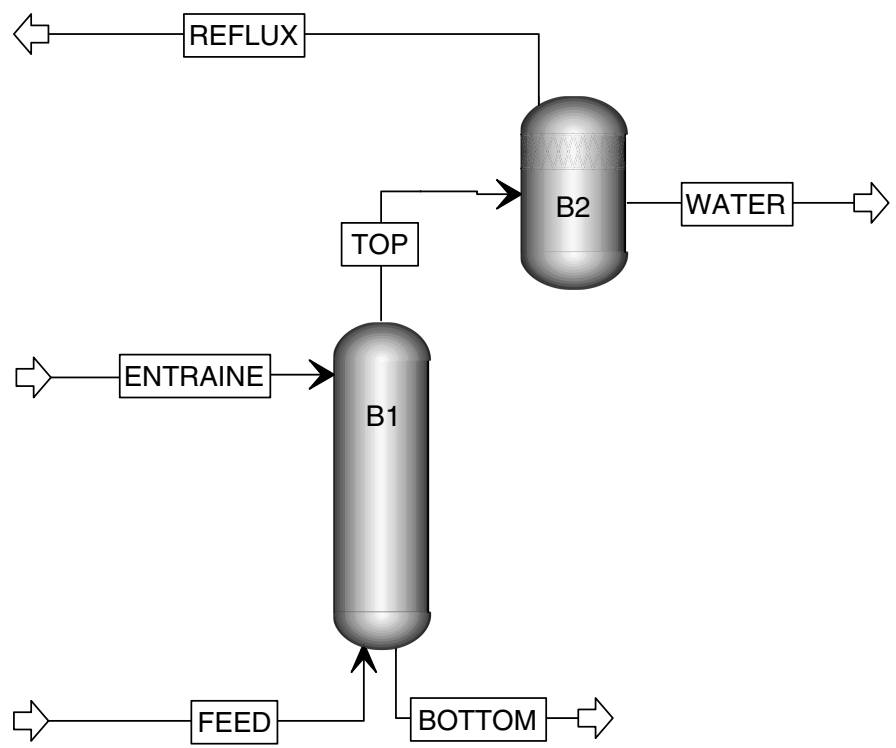

Figure 6. Flowsheet distillation section (DS)

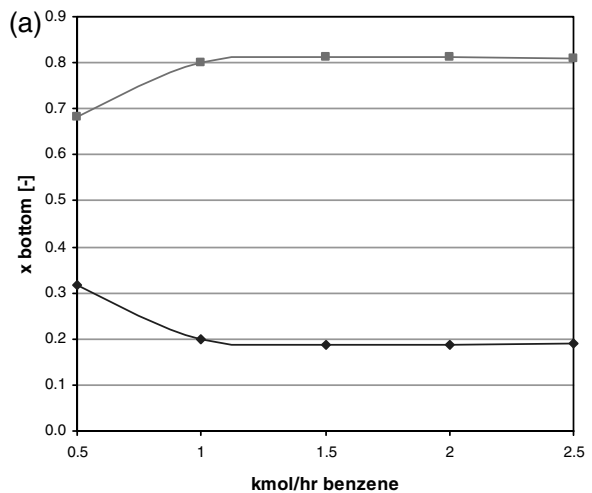

$\multimap$ water bottom $\rightarrow$ - ipa bottom

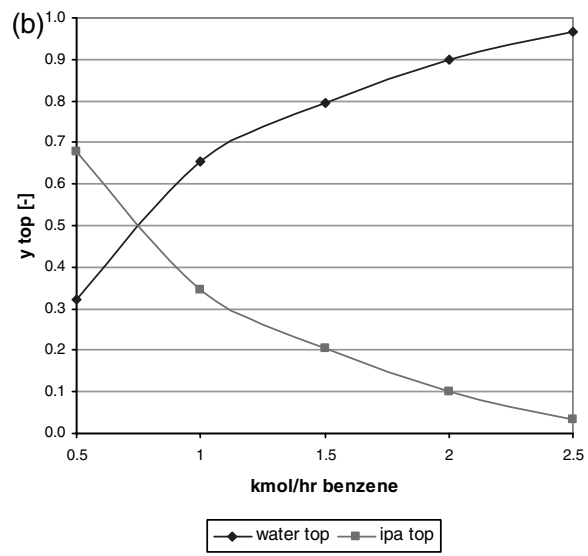

Figure 7. (a) Bottom compositions and (b) top compositions for different amounts of entrainer in a pseudo-binary mixture simulation of the distillation section (DS) 


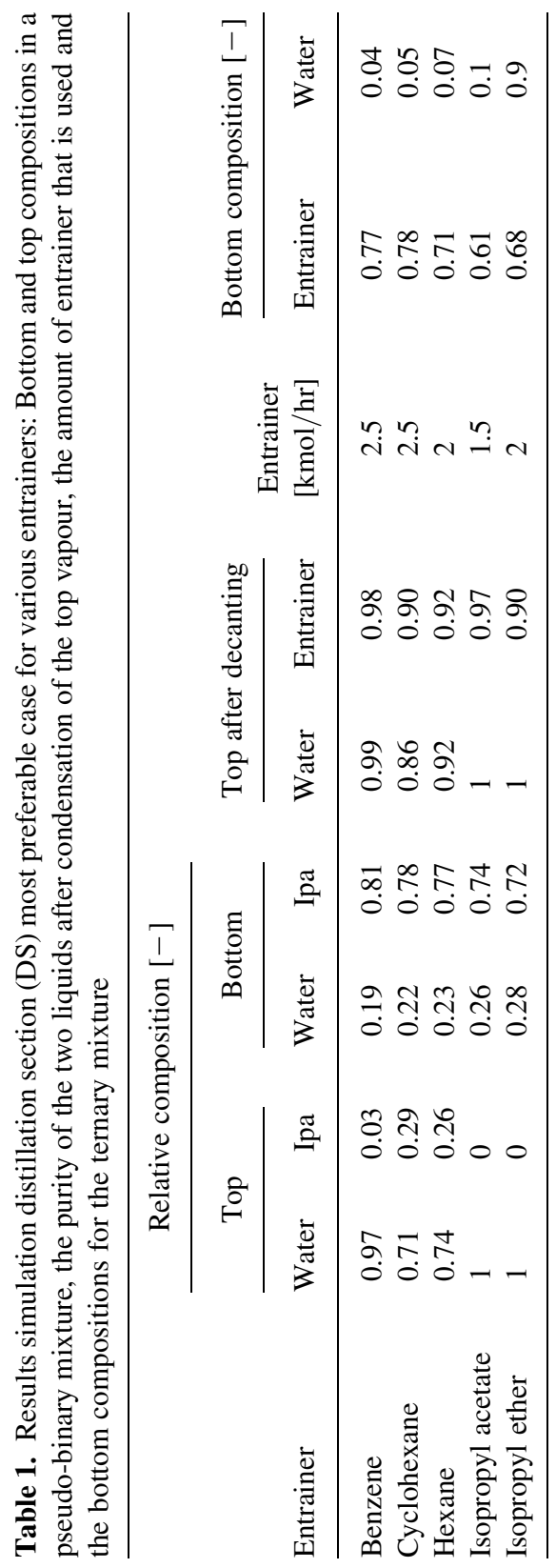


When the reflux is closed, the water-alcohol ratio in the bottom remains the same but the absolute amount of both increases as well as the amount of entrainer. The top contains more water and alcohol, and less entrainer. The water-alcohol ratio in the top decreases. This is because the reflux is not pure entrainer; it contains some water and some more alcohol.

The quantitative differences between the effects of the different entrainers are not in accordance with expectations. It is expected that the performance of removing water from the bottom increases in order of the increasing polarity: hexane $>$ cyclohexane $>$ benzene. A reason for deviations is probably that the thermodynamic data are based on Unifac Dortmund predictions instead of experimental data. Thus, reliable thermodynamic data based on experiments are necessary to quantify all effects.

\section{CONCLUSIONS}

In this paper the effect of various entrainers with different polarity on the criteria a suitable entrainer should fulfil in Entrainer-based Reactive Distillation for the esterification of a fatty acid with isopropanol was investigated. The modelling work gives good insight in what will happen if a certain entrainer is added. Based on these techniques it can be concluded that non-polar solvents are good entrainers for the studied system; they force the water out of the alcohol such that the relative volatility of water increases and they are immiscible with water such that a mixture splits in water and an entrainer phase. Thus, those two effects support each other. However, for more quantitative conclusions further research into the thermodynamics is needed. Therefore NRTL parameters will be regressed using literature data.

\section{ACKNOWLEDGEMENTS}

The authors acknowledge the financial support by STW (The Netherlands).

\section{REFERENCES}

1. Agreda, V.H., L.R. Partin, and W.H. Heise, High-purity methyl acetate via reactive distillation. Chem. Eng. Prog., 1990. February: 40-46.

2. Bock, H., G. Wozny, and B. Gutsche, Design and control of a reaction distillation column including the recovery system. Chem. Eng. Process., 1997. 36: 101-109.

3. Jeromin, L.M., N. Bremus, and E. Peukert, Kontinuierliche veresterung in Reactionskolonnen. Fette, Seifen, Anstrichmittel, 1981. 83: 493-504.

4. Schleper, B., B. Gutsche, J. Wnuck, and L. Jeromin, Einsatz eines eingachen simulationsmodells zur versuchsplanung für eine gegenstrom-veresterungskolonne. Chem. Ing. Tech., 1990. 62: 226-227.

5. Steinigeweg, S. and J. Gmehling, Esterification of a fatty acid by reactive distillation. Ind. Eng. Chem. Res., 2003. 42: 3612-3619. 
6. Omota, F., A.C. Dimian, and A. Bliek, Fatty acid esterification by reactive distillation. Part 1: equilibrium-based design. Chem. Eng. Sc., 2003. 58: 3159-3174.

7. Omota, F., A.C. Dimian, and A. Bliek, Fatty acid esterification by reactive distillation. Part 2: kinetics-based design for sulphated zirconia catalysts. Chem. Eng. Sc., 2003. 58: $3175-3185$.

8. Dimian, A.C., F. Omota, and A. Bliek, Entrainer-enhanced reactive distillation. Chem. Eng. Process., 2004. 43: 411-420.

9. Dimian, A.C., Integrated design and simulation of chemical processes. 2003, Amsterdam: Elsevier.

10. Doherty, M.F. and M.F. Malone, Conceptual design of distillation systems. 2001, Boston: McGraw-Hill.

11. Stichlmair, J.G. and J.R. Fair, Distillation principles and practice. 1998, New York: Wiley-VCH.

12. Stéger, C., V. Varga, L. Horváth, E. Rév, Z. Fonyó, M. Meyer, and Z. Lelkes, Feasibility of extractive distillation process variants in batch rectifier column. Chem. Eng. Process., 2005. 44: 1237-1256.

13. Hefter, G.T., in Hydrocarbons with water and seawater, D.G. Shaw, Editor. 1986. p. 221-229.

14. Connemann, M., J. Gaube, L. Karrer, A. Pfennig, and U. Reuter, Measurement and representation of ternary vapour-liquid-liquid equilibria. Fluid Phase Equilibria, 1990. 60: $99-118$.

15. Gomis, V., A. Font, R. Pedraza, and M.D. Saquete, Isobaric vapor-liquid and vapor-liquidliquid equilibrium data for the system water +ethanol + cyclohexane. Fluid Phase Equilibria, 2005. 235: 7-10.

16. Lee, L. and H. Shen, Azeotropic Behavior or a Water + n-Propanol + Cyclohexane Mixture Using Cyclohexane as an Entrainer for Separating the Water +n-Propanol Mixture at $760 \mathrm{~mm} \mathrm{Hg}$. Ind. Eng. Chem. Res., 2003. 42: 5905-5914.

17. Moriyoshi, T., Y. Uosaki, K. Takahashi, and T. Yamakawa, (Liquid + liquid) equilibria of (water + ethanol + cyclohexane) at the temperatures $298.15 \mathrm{~K}$ and $323.15 \mathrm{~K}$. J. Chem. Thermodynamics, 1991. 23: 37-42.

18. Plačkov, D. and I. Štern, Liquid-liquid equilibria for ternary systems of cyclohexane-water and C1 to C3 alcohols: data and predictions. Fluid Phase Equilibria, 1992. 71: 189-209.

19. Verhoeye, L.A.J., The System Cyclohexane-2-Propanol-Water. J. Chem. Eng. Data, 1968. 13(4): $462-467$. 